\title{
Theoretical Model of Diffraction Line Profiles as Combinations of Gaussian and Cauchy Distributions
}

\author{
Girija Bhushan Mitra* \\ Indian Association for the Cultivation of Science, Jadavpur, Kolkata, India \\ Email: gbmitra@gmail.com
}

Received 6 May 2014; revised 29 May 2014; accepted 17 June 2014

Copyright (C) 2014 by author and Scientific Research Publishing Inc. This work is licensed under the Creative Commons Attribution International License (CC BY). http://creativecommons.org/licenses/by/4.0/

c) (i) Open Access

\section{Abstract}

Previously we derived equations determining line broadening in ax-ray diffraction profile due to stacking faults. Here, we will consider line broadening due to particle size and strain which are the other factors affecting line broadening in a diffraction profile. When line broadening in a diffraction profile is due to particle size and strain, the theoretical model of the sample under study is either a Gaussian or a Cauchy function or a combination of these functions, e.g. Voigt and Pseudovoigt functions. Although the overall nature of these functions can be determined by Mitra's $R(x)$ test and the Pearson and Hartley $\chi$ test, details of a predicted model will be lacking. Development of a mathematical model to predict various parameters before embarking upon the actual experiment would enable correction of significant sources of error prior to calculations. Therefore, in this study, predictors of integral width, Fourier Transform, Second and Fourth Moment and Fourth Cumulant of samples represented by Gauss, Cauchy, Voigt and Pseudovoigt functions have been worked out. An additional parameter, the coefficient of excess, which is the ratio of the Fourth Moment to three times the square of the Second Moment, has been proposed. For a Gaussian profile the coefficient of excess is one, whereas for Cauchy distributions, it is a function of the lattice variable. This parameter can also be used for determining the type of distribution present in aggregates of distorted crystallites. Programs used to define the crystal structure of materials need to take this parameter into consideration.

\section{Keywords}

Line Broadening, Diffraction Profile, Theoretical Model, Gaussian Function, Cauchy Function, Voigt Function, Pseudovoigt Function, Fourier Transform, Second Moment, Fourth Moment, Fourth Cumulant

\footnotetext{
${ }^{*}$ Current Address: 284B Rash Behari Avenue, Kolkata—700019, West Bengal, India.
} 


\section{Introduction}

Diffraction line profiles due to polycrystalline materials have long been recognized to be caused by physical parameters like sizes of and defects in constituent powder particles. For this purpose, parameters like full width at half maximum (FWHM) intensity, integral width of line profile, Fourier transforms of intensity profile etc. have been utilized. Additionally, the moments [1] [2] and cumulants [3] of the line profile have also been used. These methods include recording of the line profile and correcting them for various sources of errors prior to calculations. Therefore, it may be beneficial to develop a reasonable mathematical model for the sample, the profile and the process. Such models are also required for structure refining methods as in Reitveld refinement.

The author previously showed that line broadening in a diffraction intensity profile of powdered crystalline materials due to stacking fault can be characterized in terms of the Zeroth, the First, the Second, the Third, and the Fourth Moment and the Fourth Cumulant [4]. In the present article, the scope of the characterization has been expanded to include line broadening due to particle size and strain as well. An example of a model that includes these factors may be found using Cauchy and Gauss functions. When the size effect is more predominant, the function is found to be more Cauchy-like and when the effect of strain is predominant, the Gauss model preponderates. So, the ideal model would be a suitable combination of both.

The author has in the past described a method of differentiating between Cauchy and Gauss distributions in diffraction line profiles [5]. These distributions are now standard and are used by investigators all over the world to identify the characteristics of crystalline materials. The two distributions are given as follows:

1) Gauss distribution:

$$
f_{g}(x)=\mathrm{e}^{\frac{-x^{2}}{2}}
$$

2) Cauchy distribution:

$$
f_{c}(x)=\frac{1}{1+x^{2}}
$$

where $f(x)$ is the distribution for the variable $x$. The author also showed that the cumulative distribution

$R(x)=\frac{1}{2} \int_{-x}^{x} f(x) \mathrm{d} x$ for these two distributions was provided in the past [5] as follows:

For Gauss distribution:

$$
R(x)=\frac{1}{2} \operatorname{erf} \frac{x}{\sqrt{2}}
$$

For Cauchy distribution:

$$
R(x)=\frac{1}{\pi} \tan ^{-1} x
$$

Therefore, comparison of a graph of

$$
R(x)=\int_{-x}^{x} f(x) \mathrm{d} x \quad \text { vs } x
$$

with the calculated values of

$$
\frac{1}{2} \operatorname{erf} \frac{x}{\sqrt{2}} \text { and } \frac{1}{\pi} \tan ^{-1} x,
$$

would determine the nature of the distribution, i.e., whether it is of a Cauchy or Gauss type. Using these criteria, a plot of

$$
\frac{1}{I(x)} \text { vs } x^{2}
$$

for a sample of Kaolinite, to test the formula 


$$
I(x)=\frac{1}{1+\mu^{2} x^{2}}
$$

yielded the value of $\mu$, the physical parameter describing the diffraction pattern, and revealed that the distribution in this case was of a Cauchy type [6]. On the other hand, if the material had a Gauss distribution, a plot of $\ln I(x)$ vs $\mu^{2} x^{2}$ would yield the value of $\mu$. Hence the $R(x)$ test can be used to distinguish between crystallites that are purely Gauss vs purely Cauchy.

\section{Distributions for Convolution(s) of Gauss and Cauchy Functions}

In actual practice, the distribution may be neither pure Gaussian nor pure Cauchy-but a combination of both. Several options are possible-1) a sum of partly Gaussian or partly Cauchy, 2) the convolution of partly Gaussian or partly Cauchy functions, 3) or even the same proportion of Gauss and Cauchy functions. These combinations may be purely additive or purely convolutional. The convolution may be of purely Gaussian and purely Cauchy (Voigt profile) [7] or partly Gaussian and partly Cauchy distributions (Pseudovoigt profiles) [8]. Therefore, the goal of the present effort was to identify criteria to distinguish between these various combinations. Since the convolutional combinations are likely to be the most abundant, here we shall concentrate on these criteria.

\subsection{Voigt Functions}

Convolution of two functions is by definition the inverse Fourier Transform of the product of the Fourier Transforms of the two functions i.e.

$$
F^{-1}\left\{\left(F_{g}\right) *\left(F_{c}\right)\right\}
$$

where $F_{g}$ is of the Fourier Transform of the function $g$ and $F_{c}$ is the Fourier Transform of the function $c\left(F^{-1}\right.$ is the inverse Fourier Transform of the product).

The Fourier Transform of $\mathrm{e}^{\frac{-x^{2}}{2}}$ is $\frac{1}{\sqrt{2}} \mathrm{e}^{\frac{-t^{2}}{2}}$ and Fourier Transform of $\frac{1}{1+x^{2}}=\sqrt{\frac{\pi}{2}} \mathrm{e}^{-t}$, so the product of the Fourier Transform of the function $\mathrm{e}^{\frac{-x^{2}}{2}}$ and $\frac{1}{1+x^{2}}$ is

$$
\frac{1}{\sqrt{2}} \mathrm{e}^{\frac{-t^{2}}{2}} \cdot \sqrt{\frac{\pi}{2}} \cdot \mathrm{e}^{-t}=\frac{\sqrt{\pi}}{2} \cdot \mathrm{e}^{-t^{2}-t}=\frac{\sqrt{\pi}}{2} \cdot \mathrm{e}^{-\frac{1}{4}} \cdot \mathrm{e}^{-\left(t+\frac{1}{2}\right)^{2}}
$$

Since Fourier Transform carries a function in $x$ space into $t$ space while inverse Fourier Transform takes it back into $x$ space,

$$
F^{-1}\left(\frac{\sqrt{\pi}}{2} \mathrm{e}^{-\frac{1}{4}} \mathrm{e}^{-\left(t+\frac{1}{2}\right)^{2}}\right)=\frac{\sqrt{\pi}}{2} \mathrm{e}^{-\frac{1}{4}} \mathrm{e}^{-x^{2}}, \text { using } x=t+\frac{1}{2}
$$

Thus for convolutions representing the Voigt function:

$$
f(x)=\frac{\sqrt{\pi}}{2} \mathrm{e}^{-\frac{1}{4}} \int \mathrm{e}^{-x^{2}} \mathrm{~d} x=\frac{\sqrt{\pi}}{2} \mathrm{e}^{-\frac{1}{4}} \operatorname{erf} x
$$

\subsection{Pseudovoigt Functions}

Pseudovoigt functions may be additive like $\eta$ Cauchy $+(1-\eta)$ Gauss or of convolution type like

$$
\frac{1-\eta}{\eta} \int_{0}^{x} \frac{\mathrm{e}^{-\frac{x^{2}}{2}}}{1+x^{2}} \mathrm{~d} x
$$

where $\eta$ is called the mixing fraction, and can be used to denote the proportion of intensity of the Cauchy type. 


\section{Fourier Transform of Integral Width Explaining Why Particle Size and Strain Affect Diffraction Line Profile}

\subsection{Integral Width to Express Intensity Distribution in a Diffraction Profile}

Although peak position, peak height, half intensity width etc. have been used to describe an intensity distribution in a diffraction profile, the first satisfactory parameter has been the integral width $\int I(\theta) \mathrm{d} \theta$ where $I(\theta)$ is the intensity diffracted in the direction $\theta$. For Voigt function the integral width will be

$$
\beta=\int I(x) \mathrm{d} x=\frac{\sqrt{\pi}}{2} \mathrm{e}^{-\frac{1}{4}} \int \operatorname{erf}(x) \mathrm{d} x
$$

and for the Pseudovoigt function of the additive type, this can be described in terms of

$$
\eta \tan ^{-1} x+(1-\eta) \operatorname{erf} \frac{x}{\sqrt{2}}
$$

Whereas for the Pseudovoigt function of the convolution type,

$$
\beta=\frac{\eta-1}{\eta} \frac{\sqrt{\pi}}{2} \mathrm{e}^{-\frac{1}{4}} \int \operatorname{erf}(x) \mathrm{d} x
$$

Langford introduced the Voigt function through the integral width [7]. Instead of considering the convolution of the Gauss and Cauchy functions he used the convolution of the expressions of integral width as functions of Gauss and Cauchy types-

$$
I(x)=\int_{-\infty}^{\infty} I_{c}(u) I_{g}(x-u) \mathrm{d} u
$$

Fourier Transform of $I_{c}(u)$ and $I_{g}(u)$ are given by

$$
\begin{gathered}
F_{c}(t)=\beta_{c} I_{c}(0) \exp \left(-2 \beta_{c} t\right) \\
F_{g} t=\beta_{g} I_{g}(0) \exp \left(-\pi \beta_{g}^{2} t^{2}\right) \\
F(t)=F_{c}(t) * F_{g}(t)=\beta_{c}(t) \beta_{g}(t) I_{c}(0) I_{g}(0) \exp -\left(2 \beta_{c} t+\pi \beta_{g}^{2} t^{2}\right)
\end{gathered}
$$

Thus

$$
\begin{aligned}
I(x) & =\operatorname{Re}\left(2 \beta_{c} \beta_{g} I_{g}(0) I_{c}(0) \int_{0}^{\infty} \exp -\left[\left(2 \beta_{c} i 2 \pi x\right) t+\pi \beta_{g}^{2} t^{2}\right] \mathrm{d} t\right) \\
& =\operatorname{Re}\left\{\beta_{c} I_{c}(0) I_{g}(0) \exp \left[k^{2}(1-i y)^{2}\right] \operatorname{erfc}[k(1-i y)]\right\}
\end{aligned}
$$

$\operatorname{erfc}(a-i b)$ is a complex error function defined by $\omega(z)=\exp (-t)\left[1+\frac{2 i}{\pi^{\frac{1}{2}}} \int_{0}^{z} \exp \left(t^{2}\right) \mathrm{d} t\right]$ then $I(x)=\operatorname{Re}\left\{\beta_{c} I_{c}(0) I_{g}(0) \omega\left[\pi^{\frac{1}{2}} \frac{x}{\beta_{g}}+i k\right]\right\}$.

Integral width $\beta=\frac{\beta_{g} \exp \left(-k^{2}\right)}{1-\operatorname{erfck}}$.

Ida [9] expressed the convolution in the form

$$
V(x y)=\left(\frac{y}{\pi}\right) \int_{-\infty}^{\infty} \frac{\mathrm{e}^{-t^{2}}}{(x-t)^{2}+y^{2}} \mathrm{~d} t=\operatorname{Re}[w o r f z(x+i y)]=\operatorname{Re} \exp \left(-z^{2}\right) \operatorname{erfc}(-i z)
$$

where $\operatorname{worf}(z)$ is a scaled complex error function called the Faddeva Function.

\subsection{Fourier Transform of Diffraction Profile Based on Integral Width}

As mentioned in Section 2 above, Fourier Transform of $\mathrm{e}^{\frac{-x^{2}}{2}}$ and $\frac{1}{1+x^{2}}$ are $\mathrm{e}^{-\frac{t^{2}}{2}}$ and $\frac{\sqrt{\pi}}{2} \mathrm{e}^{-t}$ respectively. 
For Voigt function the Fourier Transform is $\frac{\sqrt{\pi}}{2} \mathrm{e}^{-\frac{t^{2}}{2}-t}$.

While the Fourier Transform for Pseudovoigt function of additive type is

$$
(\eta-1) \mathrm{e}^{-\frac{t^{2}}{2}}+\eta \mathrm{e}^{-t}
$$

\subsection{Moments of Diffraction Profiles Broadened by Particle Size and Strain}

Intensity of X-ray diffracted in the direction $S=\frac{2 \sin \theta}{\lambda}$, where $\theta$ is the angle of scattering and $\lambda$ is the wave length of the radiation scattered, is given by

$$
I(s)=\int_{0}^{\tau} A(t) \exp (2 \pi i s t) \mathrm{d} t
$$

where $\tau$ is the limit where $A(t)$ vanishes. By inverse Fourier Transform

$$
A(t)=\int_{-\infty}^{\infty} I(s) \exp (2 \pi i s t) \mathrm{d} s
$$

And so, $A^{\prime}(t)=2 \pi s \int I(s) \exp (2 \pi i s t) \mathrm{d} s$, and

$$
A^{\prime}(0)=2 \pi s \int I(s) \mathrm{d} s
$$

By differentiating Equation (8) with respect to $t$ over and over again and then assuming $t=0$, we obtain the values of $A^{\prime \prime}(0), A^{\prime}(0)$ etc.

Wilson has shown that [1], the variance or the Second Moment of the diffraction profile can be given by

$$
W=-\frac{1}{4 \pi^{2}}\left[2\left(\sigma_{1}+\sigma_{2}\right) \frac{A^{\prime}(0)}{A(0)}+\frac{A^{\prime \prime}(0)}{A(0)}\right]
$$

where $\sigma_{1}$ and $\sigma_{2}$ are limits of the integral (8) instead of 0 to $\tau$ where $A(t)$ vanishes. Very often $\sigma_{1}=\sigma_{2}$.

The author [2] previously showed that the Fourth Moment of the intensity expression is given by

$$
\mu=-\frac{\sigma_{2}^{3}+\sigma_{1}^{2}}{6 \pi^{2}} \frac{A^{\prime}(0)}{A(0)}+\frac{\sigma_{1}+\sigma_{2}}{8 \pi^{4}} \frac{A^{I V}(0)}{A(0)}
$$

In Equations (11) and (12), there are other terms involving $\frac{A^{I V}(0)}{A(0)}$ in Equation (11) and $\frac{A^{I V^{\prime}}(0)}{A(0)}$ in Equation (12). But these terms are negligible compared to the remaining terms. The size of the particle comprising the powder is given by the $0^{\text {th }}$ Moment of $I(s)$ i.e.

$$
\varepsilon=\frac{1}{A(0)} \int_{-\tau}^{\tau} A(t) \mathrm{d} t
$$

The Second and the Fourth Moment of the line profile yield the shape of the particles comprising the powdered sample. Of course, they and other moments yield information regarding crystal deformations due to strain, stacking faults, nature and the extent of dislocations etc. In Equations (11)-(13) we have used equations for particle size only, disregarding other factors like strain, dislocation density etc.

The author previously also showed that for a powder particle of size $p_{1}, p_{2}$ and $p_{3}$ with interfacial angles $\alpha, \beta$ and $\gamma$ and Lattice constants $a, b, c$, the volume common to the crystal and its ghost removed by $t$ in the $h k l$ direction, is given by [6]

$$
A(t)=\left(p_{1}-h^{\prime} t\right)\left(p_{2}-k^{\prime} t\right)\left(p_{3}-l^{\prime} t\right) \phi
$$

where $h^{\prime}=\frac{h d}{a}, k^{\prime}=\frac{k d}{b}$ and $l^{\prime}=\frac{l d}{c}, d$ is the interplanar spacing in the direction of diffraction and

$$
\phi=\sqrt{1-\cos ^{2} \alpha-\cos ^{2} \beta-\cos ^{2} \gamma+2 \cos \alpha \cos \beta \cos \gamma}
$$


where $\alpha, \beta$ and $\gamma$ are angles between the axes

Thus $A(t)=\left[p_{1} p_{2} p_{3}-\left(p_{1} p_{2} k^{\prime}+p_{2} p_{3} l^{\prime}+p_{2} p_{3} h^{\prime}\right) t+\left(p_{3} h^{\prime} k^{\prime}+p_{1} k^{\prime} l^{\prime}+p_{2} h^{\prime} l^{\prime}\right) t^{2}-h^{\prime} k^{\prime} l^{\prime} t^{3}\right] \phi$

Therefore,

$$
\begin{gathered}
A(0)=p_{1} p_{2} p_{3} \phi \\
A^{\prime}(0)=-\left(p_{1} p_{3} k^{\prime}+p_{2} p_{1} l^{\prime}+p_{3} p_{1} h^{\prime}\right) \phi \\
A^{\prime \prime}(0)=2\left(p_{3} h^{\prime} k^{\prime}+p_{1} k^{\prime} l^{\prime}+p_{2} h^{\prime} l^{\prime}\right) \phi \\
A^{\prime \prime \prime}(0)=3 h^{\prime} k^{\prime} l^{\prime} \phi
\end{gathered}
$$

Hence

$$
\begin{gathered}
\frac{A^{\prime}(0)}{A(0)}=-\left(\frac{k^{\prime}}{p_{2}}+\frac{l^{\prime}}{p_{3}}+\frac{h^{\prime}}{p_{2}}\right) \\
\frac{A^{\prime \prime}(0)}{A(0)}=2\left(\frac{h^{\prime} k^{\prime}}{p_{1} p_{2}}+\frac{k^{\prime} l^{\prime}}{p_{2} p_{3}}+\frac{h^{\prime} l^{\prime}}{p_{1} p_{3}}\right) \\
\frac{A^{\prime \prime \prime}(0)}{A(0)}=3 \frac{h^{\prime} k^{\prime} l^{\prime}}{p_{1} p_{2} p_{3}}
\end{gathered}
$$

For $h 00,0 k 0$ and $00 l$ reflection, only one dimension of the particle will be obtained, with $h k 0,0 k l$ and $h 0 l$ reflections only two dimensions will be obtained. For a cylindrical crystal, the above equations will be modified in terms of equations derived by Langford and Louër [10].

\section{Moments of Different Distributions}

\subsection{The Gaussian Profile}

It is known from Equation 509 [11] that

$$
\int_{0}^{\infty} x^{2 n} \cdot \mathrm{e}^{-a x^{2}} \mathrm{~d} x=\frac{1 \cdot 3 \cdot 5 \cdots(2 n-1)}{2^{n+1} \cdot a^{n}} \sqrt{\frac{\pi}{a}}
$$

Putting $n=1$ and $a=1 / 2$, we have the expression for the Second Moment of a Gaussian function,

$$
\int x^{2} \cdot \mathrm{e}^{-\frac{x^{2}}{2}} \mathrm{~d} x=\frac{1}{2} \sqrt{2 \pi}
$$

And putting $n=2$ and $a=1 / 2$, we have the expression for the Fourth Moment of a Gaussian function

$$
\int x^{4} \cdot \mathrm{e}^{-\frac{x^{2}}{2}} \mathrm{~d} x=\frac{3}{2} \sqrt{2 \pi}
$$

Equations (15) and (16) represent the Second and the Fourth Moment of a Gaussian profile. Cernansky has derived the equations [3]

$$
\mu_{g}^{r}=\int_{-\infty}^{\infty} \exp \left(-r^{2} s^{2}\right) \mathrm{d} s=\frac{\sqrt{\pi}}{r} \text { and } \mu_{2 g}^{r}=\frac{1}{\mu_{0}} \int_{-\infty}^{\infty} s^{2} \exp \left(-r^{2} s^{2}\right) \mathrm{d} s=\frac{1}{2 r^{2}}
$$

and

$$
\mu_{4 g}^{r}=\frac{1}{\mu_{0}} \int_{-\infty}^{\infty} s^{4} \exp \left(-r^{2} s^{2}\right) \mathrm{d} s=\frac{3}{4 r^{4}}
$$

\subsection{The Cauchy Profile}

For a Cauchy profile $f(x)=\frac{1}{1+x^{2}}$, the Second Moment, using Equation 67 [11], is 


$$
\int_{-n}^{+n} \frac{x^{2} \mathrm{~d} x}{1+x^{2}}=x-\tan ^{-1} x
$$

and the Fourth Moment, from Equation 2.147.3 [10], is

$$
\int_{-n}^{+n} \frac{x^{4} \mathrm{~d} x}{1+x^{2}}=\frac{x^{3}}{3}-x+\tan ^{-1} x
$$

\subsection{The Voigt Profile}

The Second Moment of the Voigt profile is given by

$$
x^{2} I(x)-2 x \int I(x) \mathrm{d} x
$$

where $I(x)=\frac{\sqrt{\pi}}{2} \mathrm{e}^{-\frac{1}{4}} \operatorname{erf} x$ from Equation (7a)

$$
\begin{aligned}
& =\frac{\sqrt{\pi}}{2} \mathrm{e}^{-\frac{1}{4}} \cdot \frac{2}{\sqrt{\pi}}\left[x-\frac{x^{3}}{3}+\frac{x^{5}}{10}\right] \text { from Equation } 811[11] \\
& =\mathrm{e}^{-\frac{1}{4}}\left[x-\frac{x^{3}}{3}+\frac{x^{5}}{10}\right] .
\end{aligned}
$$

For large positive value of $x$

$\operatorname{erf} x=1-\frac{\exp \left(-x^{2}\right)}{x \sqrt{\pi}}\left[1-\frac{1}{2 x^{2}}+\frac{3}{4 x^{3}}\right]$ from Equation 812 [11].

Similarly the Fourth Moment of the Voigt profile is given by

$$
\int x^{4} \frac{\mathrm{e}^{-\frac{x^{2}}{2}}}{1+x^{2}} \mathrm{~d} x=x^{4} I(x)-4 x^{3} \int I(x) \mathrm{d} x+12 x^{2} \iint I(x) \mathrm{d} x \mathrm{~d} x
$$

\subsection{The Pseudovoigt Profile}

For the Pseudovoigt profile of the convolution type the Second and Fourth Moment will be given by Equations (19) and (20) multiplied by $\frac{1-\eta}{\eta}$ and for Pseudovoigt profiles of additive type

1) Second Moment

$$
(1-\eta) \frac{1}{2} \sqrt{2 \pi}+\eta\left(x-\tan ^{-1} x\right)
$$

2) Fourth Moment

$$
(1-\eta) \frac{3}{2} \sqrt{2 \pi}+\eta\left[\frac{x^{3}}{3}-x+\tan ^{-1} x\right]
$$

\section{The Fourth Cumulant for the Different Distributions}

Since the Second Moment and Second Cumulant are equal and we have already studied the Second Moment, we shall be examining only the Fourth Cumulant. All cumulants along with the Fourth Cumulant have the additive property namely

$$
C_{4}\left(x_{1}+x_{2}+x_{3}+\cdots\right)=C_{4}\left(x_{1}\right)+C_{4}\left(x_{2}\right)+C_{4}\left(x_{3}\right)+\cdots
$$

$x_{1}, x_{2}, x_{3}$ being specific properties like size, strain, stacking fault, probability etc. The Fourth Cumulant $C_{4}$ is given by

$$
C_{4}=\mu_{4}-3 \mu_{2}^{2}
$$


where $\mu_{4}$ and $\mu_{2}$ are the Fourth and Second Moment respectively which have been described in this paper. The Fourth Cumulant is given as shown below.

\subsection{For Gaussian Distribution}

Fourth Cumulant $C_{4 g}=\mu_{4 g}-3 \mu_{2 g}^{2}$

$$
\begin{aligned}
& \mu_{4 g}=\frac{3}{2} \sqrt{2 \pi} \text { by Equation (16) } \\
& \mu_{2 g}=\frac{1}{2} \sqrt{2 \pi} \text { by Equation (15) }
\end{aligned}
$$

$$
C_{4 g}=\frac{3}{2} \sqrt{2 \pi}-\frac{3}{2} \pi=\frac{3}{2}(\sqrt{2 \pi}-\pi)
$$

according to Cernansky $C_{4 q}=0$ [3]. Indeed, Equations (22) and (23) indicate that it is a very small quantity.

\subsection{For Cauchy Distribution}

By Equation (18) $\mu_{4 c}=\frac{x^{3}}{3}-x+\tan ^{-1} x$

By Equation (17) $\mu_{2 c}=x-\tan ^{-1} x$

Thus

$$
C_{4 c}=\mu_{4 c}-3 \mu_{2 c}^{2}=\frac{x^{3}}{3}-4 x+\tan ^{-1} x\left(7-3 \tan ^{-1} x\right)
$$

\subsection{For Voigt Distribution}

$C_{4 v}=\mu_{4 v}-3 \mu_{2 v}^{2}$ from Equations (19) and (20).

\subsection{For Pseudovoigt Distributions}

The Fourth Cumulant can be found similarly from Equations (21) and (22). Now, to distinguish between Voigt and Pseudovoigt functions, an additional parameter, the coefficient of excess, is introduced here. It is the ratio of the Fourth Moment and three times the square of the Second Moment [12]. According to Cernansky for a Gaussian system the ratio is 1 [3]. The above calculations show that for the Cauchy system, the ratio is $\frac{\mu_{4 c}}{3 \mu_{2 c}^{2}}=\frac{x^{3}-3 x+3 \tan ^{-1} x}{9\left[x^{2}-2 x \tan ^{-1} x\left(1-\tan ^{-1} x\right)\right]}$ from Equations (18) and (17) respectively.

Hence this study proposes that Equations (19) and (21) may be used for identifying Voigt and Pseudovoigt distributions, respectively.

\subsection{The $\chi$ Test}

Pearson and Hartley [13] have described the $\chi$ test for determining the nature of the lineprofile. Here $\chi=\frac{\mu_{4}}{\mu_{2}}=\frac{3}{2} r^{2}$ for Gaussian function and $\frac{x^{3}-x+\tan ^{-1} x}{x-\tan ^{-1} x}$ for Cauchy distribution.

For Voigt and Pseudovoigt distribution Equations (20) and (19), (22) and (21) should be used.

\section{Parameters from Either a Single or a Few Lines}

\subsection{The Method of Line Breadth}

That more than one parameter can be studied from one line in a Debye Scherrer pattern was noticed by Williamson \& Hall who assumed that particle size and strain broadenings had Cauchy like distribution [14]. This 
means that the observed breadth of a line $\beta$ is the sum of breadths due to particle size and strain i.e.

$$
\beta_{\text {obs }}=\beta_{\text {particlesize }}+\beta_{\text {strain }}
$$

or

$$
\beta=\frac{\lambda}{t \cos \theta}+2 e \tan \theta
$$

where $t$ is the apparent particle size and $e$ is apparent strain, $\theta$ is the angle of scattering and $\lambda$ is the wave length scattered.

Thus

$$
\frac{\beta \cos \theta}{\lambda}=\frac{1}{t}+2 e \sin \theta
$$

Plotting $\frac{\beta \cos \theta}{\lambda}$ vs $\sin \theta$, one can obtain the particle size $t$ from the intercept one from the slope of the curve. Equation (25) is the well-known Williamson-Hall relation much used by many investigators [14]. Particle size and strain also can be determined by the famous Warren-Averbach method [15].

\subsection{The Method of Line Profile}

Warren and Averbach showed that the Fourier Transform of a line profile yield significant information regarding particle size, particle strain, probability of faulting and many other crystal defects [15]. They also showed that an intensity profile can be expressed as a Fourier series

$$
I(s)=\int v(t) \exp 2 \pi i s t \mathrm{~d} t
$$

where $v(t)$ is the Fourier Transform of the particle size, (st) stands for strain. For determining particle size and strain only, Warren and Averbach has developed a simple method [15]. Convert hkl reflection into $h_{1} k_{1} l_{1}$, $2 h_{1} 2 k_{1} 2 l_{1}$ etc. reflections by suitably changing axes as far as possible. Then let them be considered as 00l, 002l, 0031 etc. by necessarily changing axes. Then write

$$
I(s)=\sum_{n} A_{n}(l) A_{n}(d) \cos 2 \pi s t=\sum_{n} A_{n}(l) A_{n}(d) \cos 2 \pi l Z_{n}
$$

Now

$$
\cos \left\langle 2 \pi l Z_{n}\right\rangle=l_{n}\left(1-2 \pi^{2} l^{2}\left\langle Z_{n}\right\rangle^{2}\right)=-2 \pi^{2} l^{2}\left\langle Z_{n}\right\rangle^{2}
$$

For small value of $l$ and $n$, the logarithm of the measured Fourier coefficient is given by

$$
l_{n} A_{n}(l)=l_{n} A_{n}^{s}-2 \pi^{2} l^{2}\left\langle Z_{n}^{2}\right\rangle
$$

By plotting $l_{n} A_{(n)}$ against $l^{2}$ we obtain at $l=0$, the value of $A_{n}^{s}$ and from the slope, $-2 \pi^{2}\left\langle Z_{n}^{2}\right\rangle$. The distance $l=n a_{3}$ is the undistorted distance $a_{3} Z_{n}$.

In general, Equation (26) can be written in a summation form

$$
I(s)=\sum_{n}\left[A_{n} \cos 2 \pi n s t+B_{n} \sin 2 \pi n s t\right]
$$

where $A_{n}=A_{n}^{d} \cdot A_{n}^{s}, A_{n}^{d}$ is the coefficient for particle size broadening, $A_{n}^{s}$ for strain. Warren and Warekois [16] showed that this causes a shift in peak position in $70.50 \alpha$ brass and $\mathrm{CoK}_{\alpha}$ radiation.

$$
\Delta\left(2 \theta_{200}-2 \theta_{111}\right)^{\circ}=-6.2 p
$$

where $p$ is the stalking fault probability. Also

$$
-\left(\frac{\mathrm{d} A_{n}^{\mathrm{s}}}{\mathrm{d} n}\right)_{111}=\frac{1}{D}+\frac{1.5 \alpha+\gamma}{a} \frac{\sqrt{3}}{4}
$$

And 


$$
-\left(\frac{\mathrm{d} A_{n}^{\mathrm{s}}}{\mathrm{d} n}\right)_{200}=\frac{1}{D}+\frac{1.5 \alpha+\gamma}{a}
$$

where $\gamma$ is the twin fault probability, and $a$ is the lattice constant.

\section{Conclusion}

The application of the above formulae depends on the recognition of the proper mathematical model. To achieve this, the best way is the $R(x)$ test devised by Mitra [5] to distinguish between Gauss and Cauchy profiles (described in [13]). Plot of intensity vs the angle of curve will yield considerable information. For each peak-nonoverlapped by neighboring peaks, the corresponding intensity vs angle of scattering curve should be examined to determine if it belongs to Gaussian, Cauchy, Voigt and Pseudovoigt type and if so, the mixing parameter can be obtained by comparing with erf $\frac{x}{\sqrt{2}}, \tan ^{-1} x$, erf $x, \frac{1-\eta}{\eta} \operatorname{erf} x,(1-\eta) \operatorname{erf} \frac{x}{\sqrt{2}}$ and $(1-\eta) \operatorname{erf} \frac{x}{\sqrt{2}}+\eta \tan ^{-1} x$ respectively. There is a likelihood of confusion between Gaussian, Voigt and Pseudovoigt of convolution type. This can probably be resolved by examining additional peaks. Similarly, determining particle sizes by trying the relevant formulae may be helpful. The advantage of this procedure is that overlapping of lines will not interfere with the determination of the parameters. It is important to note that the Fourth Cumulant is an important parameter-since it is the only parameter which can decompose the observed line profile into profiles for different reasons of line shape. By a reverse analysis of the shape of the diffraction profile, it should be possible to identify the nature of the line profile—Gauss, Cauchy, Voigt, Pseudovoigt and hence, of the mixing parameter. From these-it should be possible to determine the parameters of line broadening. It is expected to be a good test for pattern decomposition. In this connection, another test-the $\chi$ test due to Pearson and Hartley may be mentioned [13]. $\chi=\frac{\mu_{4}}{\mu_{2}}$, and this can be calculated for the Gauss, Cauchy, Voigt and Pseudovoigt functions using Formulae (15), (17) and (18). Pearson and Hartley have given numerical values to identify Pearson type IV and Pearson type VII curves as well [13].

\section{Acknowledgements}

Sincere and heartfelt thanks are due to Dr. Paramita M Ghosh of the University of California at Davis, California, USA for encouragement and helping in many ways and to Mr. Bishwajit Halder for secretarial help. The author was previously Professor and Head of the Department of Physics, Indian Institute of Technology, Kharagpur, West Bengal, INDIA.

\section{References}

[1] Wilson, A.J.C. (1962) Refraction Broadening in Powder Diffractometry. Proceedings of the Physical Society, 80, 303305. http://dx.doi.org/10.1088/0370-1328/80/1/134

[2] Mitra, G.B. (1964) The Fourth Moment of Diffraction Profiles. British Journal of Applied Physics, 15, 917-921. http://dx.doi.org/10.1088/0508-3443/15/8/305

[3] Cernanský, M. (2008) Cumulants and Moments in the Line Profile Analysis. Zeitschrift für Kristallographie Supplements, 27, 127-133. http://dx.doi.org/10.1524/zksu.2008.0017

[4] Mitra, G.B. (2013) Moments and Cumulants of Diffraction Profiles Broadened by Stacking Faults. Journal of Crystallization Process and Technology, 3, 103-107. http://dx.doi.org/10.4236/jcpt.2013.33017

[5] Mitra, G.B. (1963) A Method of Distinguishing between Gauss and Cauchy Diffraction Profiles. Acta Crystallographica, 16, 429. http://dx.doi.org/10.1107/S0365110X63001110

[6] Mitra, G.B. (1963) Structure Defects in Kaolinite. Zeitschrift für Kristallographie, 119, 161-175. http://dx.doi.org/10.1524/zkri.1963.119.3-4.161

[7] Langford, J. (1978) A Rapid Method for Analysing the Breadths of Diffraction and Spectral Lines Using the Voigt Function. Journal of Applied Crystallography, 11, 10-14. http://dx.doi.org/10.1107/S0021889878012601

[8] Sanchez-Bajo, F. and Cumbrera, F.L. (1997) The Use of the Pseudo-Voigt Function in the Variance Method of X-Ray 
Line-Broadening Analysis. Journal of Applied Crystallography, 30, 427-430. http://dx.doi.org/10.1107/S0021889896015464

[9] Pierce, B.O. and Foster, R.M. (1966) A Short Table of Integrals: Fourth Edition. 4th Edition, Blaisdell Publishing Co., New York, 96.

[10] Langford, J.I. and Louer, D. (1982) Diffraction Line Profiles and Scherrer Constants for Materials with Cylindrical Crystallites. Journal of Applied Crystallography, 15, 20-26. http://dx.doi.org/10.1107/S0021889882011297

[11] Pierce, B.O. and Foster, R.M. (1966) A Short Table of Integrals Vol. 298. Blaisdell, Waltham, 43.

[12] Aitken, A.C. (1957) Statistical Mathematics. Oliver and Boyd, London.

[13] Pearson, E.S. and Hartley, H.O. (1956) Biometric Tables for Statisticians Vol. 1. Cambridge University Press, London, 210.

[14] Williamson, G.K. and Hall, W.H. (1953) X-Ray Line Broadening from Filed Aluminium and Wolfram. Acta Metallurgica, 1, 22-31. http://dx.doi.org/10.1016/0001-6160(53)90006-6

[15] Gradshteyn, I.S. and Ryzhik, I.M. (1980) Table of Integrals, Series and Products. 4th Edition, Academic Press, Inc., Orlando, 1160.

[16] Mittemeijer, E.J. and Welzel, U. (2008) The "State of the Art" of the Diffraction Analysis of Crystallite Size and Lattice Strain. Zeitschrift für Kristallographie, 223, 552-560. http://dx.doi.org/10.1524/zkri.2008.1213 
Scientific Research Publishing (SCIRP) is one of the largest Open Access journal publishers. It is currently publishing more than 200 open access, online, peer-reviewed journals covering a wide range of academic disciplines. SCIRP serves the worldwide academic communities and contributes to the progress and application of science with its publication.

Other selected journals from SCIRP are listed as below. Submit your manuscript to us via either submit@scirp.org or Online Submission Portal.
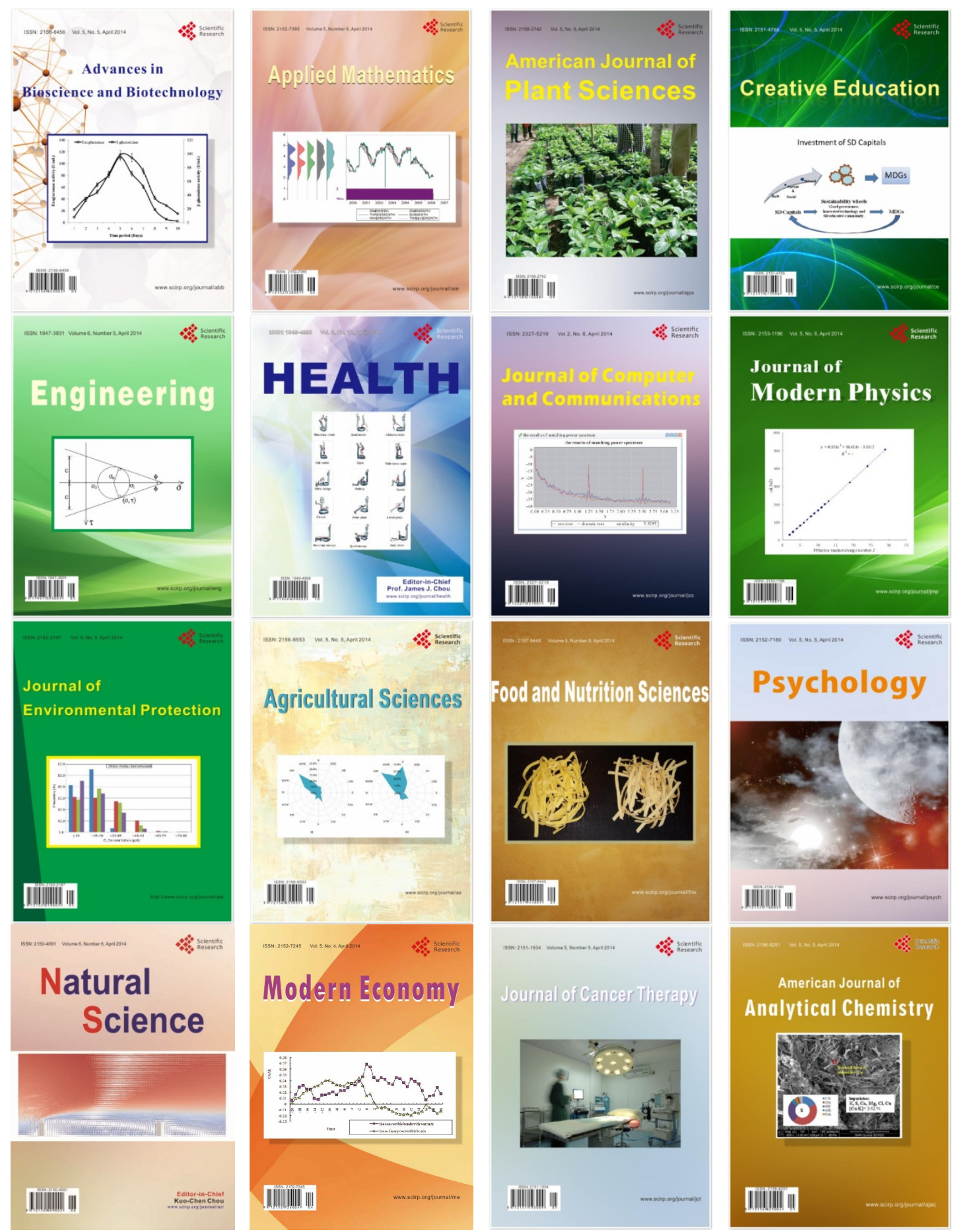